\title{
Association of parental pretransplant psychosocial assessment with post-transplant morbidity in pediatric heart transplant recipients*
}

Stone D, Banerjee M, Dupuis J, Leleszi J, Allasio D, Singh TP. Association of parental pretransplant psychosocial assessment with post-transplant morbidity in pediatric heart transplant recipients. Pediatr Transplantation 2006: 10:602-607. (C) 2006 Blackwell Munksgaard

Abstract: Because parents assume the primary responsibility for providing ambulatory post-transplant care to pediatric patients, pretransplant psychosocial evaluation in these recipients is usually focused on parents rather than on patients themselves. We sought to determine whether pretransplant parental psychosocial evaluation predicts posttransplant medical outcome at current levels of psychosocial support. We compared relative risk (RR) of rejection and hospitalizations (days of all-cause hospitalization) following initial discharge in patients in 'risk' and 'control' groups defined by their pretransplant parental psychosocial evaluation. We also compared the two groups of patients for the proportion of all outpatient trough cyclosporine A (CSA) or tacrolimus (FK) levels that were $<50 \%$ of the target level (defined as the mid-therapeutic range level). There were seven patients in the 'risk' group with a median age $0.25 \mathrm{yr}$ (range $0.19-14.7 \mathrm{yr}$ ) and total follow up 20.5 patient-yr. There were 21 patients in the 'control' groups with a median age of $2.1 \mathrm{yr}$ (range $0.05-16.2 \mathrm{yr}$ ) and total follow up of 71.3 patient-yr. There was no significant difference between the groups in rejection-risk or days of all-cause hospitalization early after transplant (first six months). During the late period (after the first six months), there were 11 rejection episodes in the 'risk' group over 17.4 patient-yr and four rejection episodes in control group over 61.8 patient-yr of follow up. After adjustment for age and race, patients in the 'risk' category had a RR of 3.4 for developing a rejection episode $(p=0.06)$ and 3.1 for being inpatient $(p<0.001)$ during the late period. Patients in the risk group were 2.9 times more likely to have subtherapeutic trough levels ( $<50 \%$ target level) of calcineurin inhibitor (CSA or FK) during both early and late periods ( $p<0.01$ for both periods) after adjustment for patient age and race. We conclude that pretransplant parental psychosocial risk assessment is associated with post-transplant morbidity in children after cardiac transplantation. These patients may benefit from closer outpatient monitoring and a higher level of psychosocial support.

\author{
David Stone', Mousumi Banerjee ${ }^{2}$, \\ Joanne Dupuis ${ }^{1}$, Jimmie Leleszi ${ }^{1}$, \\ David Allasio $^{1}$ and Tajinder P. Singh ${ }^{1}$ \\ ${ }^{1}$ Department of Pediatrics, Children's Hospital of \\ Michigan, Wayne State University School of \\ Medicine, Detroit, MI, '2Department of Biostatistics, \\ School of Public Health, University of Michigan, Ann \\ Arbor, MI, USA
}

Key words: transplantation - psychosocial pediatric - rejection

\footnotetext{
* Presented in part during the scientific sessions of the International Society for Heart and Lung Transplantation, Philadelphia, PA, April 2005 and during the scientific sessions of American Heart Association, Dallas, TX, November 2005.

Tajinder P. Singh, Heart Transplant Program, Department of Cardiology, Children's Hospital, 300 Longwood Avenue, Boston, MA 02115, USA Tel.: +1 6173556329

Fax: +1 6177349930

E-mail: tp.singh@cardio.chboston.org

Accepted for publication 4 April 2006
}

In patients with severe congenital or acquired heart disease, OHT often provides the only opportunity for survival. Post-transplant patient

Abbreviations: CI, calcineurin inhibitors; CSA, cyclosporine A; FK, tacrolimus; NS, not significant; OHT, orthotopic heart transplantation; RR, relative risk. survival continues to improve; the current one-yr survival approaches $90 \%(1,2)$. Previous studies have identified black race and teenage age to be independent risk factors for recurrent rejection (3). A higher rejection-risk in black recipients may be explained in part by immunologic factors (4). However, socioeconomic, demographic and 
psychosocial factors that influence access to healthcare resources as well as patient compliance following transplant may also be important determinants of patient outcome following transplant. Indeed, psychosocial evaluations that assess these factors appear to be useful in identifying adult OHT recipients at higher risk of adverse outcome (5-7). Psychosocial evaluation of the recipient is routinely performed at most centers prior to listing for OHT and is considered a requirement by third party payers. This evaluation identifies the challenges likely to be faced by the patient, the family and the transplant team following OHT thus allowing focused intervention and additional psychosocial support if necessary. Because parents assume primary responsibility for providing ambulatory post-transplant care to pediatric patients, pretransplant psychosocial evaluation for pediatric recipients is usually focused on parents rather than on patients themselves. Parental noncompliance has been found to be a major issue affecting outcome following organ transplantation in pediatric patients. Specifically, noncompliance was demonstrated to comprise the majority of problems in pediatric patients following liver transplantation, including dysfunction, hospitalization and graft loss (8). We have observed that patients for whom no psychosocial concerns are raised prior to transplant by either a formal evaluation or by nurses caring for them are less likely to have recurrent rejection and are less likely to require hospitalization for medical morbidity after their initial post-transplant discharge. We hypothesized that children whose parents are identified to be 'at-risk from a psychosocial perspective' will be at a higher risk of recurrent rejection compared with controls and will have a higher incidence-rate of days of all-cause hospitalization following their initial discharge following OHT. We also compared the risk of low trough levels of CI between the two patient groups classified according to their psychosocial risk.

\section{Patients and methods}

This was a single-center retrospective cohort study conducted after obtaining approval from institutional Human Investigation Committee. The study included all 28 patients $<18 \mathrm{yr}$ of age who underwent cardiac transplantation at our institution since initiation of the transplant program in 1998 and who survived to discharge following OHT. Of these, 15 patients were male $(54 \%)$ and 13 were female $(46 \%)$. Thirteen patients were black $(46 \%), 12$ Caucasian $(43 \%)$, two Hispanic $(7 \%)$ and one Asian (4\%). The indication for cardiac transplantation was un-repaired congenital heart disease in $14(50 \%)$, and cardiomyopathy in nine patients $(32 \%)$. Five patients $(18 \%)$ had previously undergone a palliative procedure for congenital heart disease.

\section{Psychosocial categories}

A pediatric psychiatrist and a social worker with experience in evaluating organ transplant candidates assigned each patient into 'risk' and 'control' groups after reviewing pretransplant psychosocial characteristics obtained from the pretransplant social work and psychiatry consultations from patient medical records. A psychosocial risk stratification inventory was supplied as a guideline for categorization (6). This reference placed patients into one of three categories based on their psychosocial risk. The data collected for this study included parental age, education, employment, marital status, family size, means of transportation to the hospital, support systems, religious beliefs regarding organ transplantation, past history of protective service investigations, substance abuse, domestic violence, noncompliance, psychiatric illness and case referral to the ethics committee. The assigners were blinded to patient identifiers, race and medical outcome and classified patients based on their concerns regarding the parent's ability to care for a medically complex patient. Due to a small sample size $(\mathrm{n}=$ 28), two (control and risk groups) rather than three psychosocial risk categories were defined. The social worker and psychiatrist agreed on the risk assignment in all 28 patients.

\section{Outcome variables}

We evaluated two primary outcome variables in this study: (i) time to next rejection episode; and (ii) days of all-cause hospitalization following the initial post-transplant discharge. Because the risk of these outcomes is different early (first few months) and late after transplant, we evaluated these variables and their predictors separately for the first six-month period (early period) and after the six months (late period) following initial patient discharge. We defined a rejection episode as any event that led to enhanced immune suppression as previously defined in studies published by the Pediatric Heart Transplant Study Group (3). This enhanced immune suppression usually followed a diagnosis of rejection by clinical and echocardiography findings often with additional confirmation with an endomyocardial biopsy. On other occasions, rejection was diagnosed by histopathologic findings following a routine surveillance endomyocardial biopsy. We also compared the two groups of patients for the proportion of all outpatient trough CSA or FK levels that were $<50 \%$ of the target level (defined as the mid-therapeutic range level) that could not be explained by an intercurrent illness (such as gastroenteritis), drug interaction (recent medication change) or a delayed blood draw.

\section{Statistical analysis}

The RR for rejection was analyzed using a marginal Cox proportional hazards model for recurrent events which allowed us to account for all rejection episodes in each patient. Estimates of the RR (hazard ratio) for the 'risk' group were calculated for early and late time periods using univariate as well as multivariable analyses, and 95\% confidence intervals constructed using robust sandwich variance estimate. In the multivariable analyses, we adjusted for two previously described risk factors - patient race (black vs. non-black) and age ( $>10 \mathrm{vs.} \leq 10 \mathrm{yr}$ ). The RR (incidence 
Stone et al.

rate ratio) of being inpatient (days of all-cause hospitalization) for the 'risk' group and the corresponding 95\% confidence intervals were calculated for both time periods using Poisson regression models. Logistic regression was used to compare the proportion of low CSA or FK levels between the 'risk' and 'control' groups, adjusting for patient race and age in a multivariable analysis as above.

\section{Results}

Of the 28 patients, seven patients were assigned to the psychosocial 'risk' group and 21 patients to the 'control' group. The median age at transplant in the 'risk' group was $0.25 \mathrm{yr}$ (range 0.19$14.7 \mathrm{yr}$ ). Their median follow up in this group after initial discharge was 913 days (range 28-2878 days). This resulted in a total of 20.5 patient-yr of follow up. The median age at transplant in the 'control' group was $2.1 \mathrm{yr}$ (range 0.05-16.2 yr). Their median follow up after OHT discharge was 1256 days (range 25-2484 days). This resulted in a total of 71.3 patient-yr of follow up in controls. There was one death in the 'risk' group and one patient left our institution two yr following OHT. There were no deaths or loss to follow up in controls.

\section{Number of rejection episodes}

During the early period (first 6 months), there were three rejection episodes in the 'risk' group over 3.0 patient-yr of follow up and eight rejection episodes in control group over 8.9 patient-yr of follow up. This resulted in incidence rates of rejection of 1.0/patient-yr in the 'risk' group and $0.9 /$ patient-yr in the control group during this period $(\mathrm{p}=\mathrm{NS}$, incidence rate ratio $=1.07)$.
During the late period (after the first 6 months), there were 11 rejection episodes in the 'risk' group over 17.4 patient-yr of follow up and four rejection episodes in control group over 61.8 patient-yr of follow up. This resulted in incidence rates of rejection of $0.63 /$ patient-yr in the 'risk' group and $0.05 /$ patient-yr in the 'control' group during this period $(\mathrm{p}<0.001$, incidence rate ratio $=12.6$ ).

Time to rejection

During the early period (first six months), black race was the only significant predictor for rejection $(\mathrm{p}=0.04$ in the univariate analysis and 0.06 in the multivariable analysis) (Table 1). The psychosocial 'risk' category was not a predictor for rejection for this period. For the late period, patient age $(>10 \mathrm{yr})$, black race and psychosocial 'risk' group were all associated with rejection in the univariate analysis. After adjustment for patient age and race in a multivariable analysis, patients in the 'risk' category had a RR of 3.4 for rejection compared with patients in the control group $(\mathrm{p}=0.06)$.

\section{All-cause hospitalization}

The length of all-cause hospital stay during the early period (six months following initial discharge) was 20.1 days/patient-yr in the 'risk' group compared with 13.5 days/patient-yr in controls $(p=0.01)$ (Table 1$)$. The length of allcause hospital stay during the late period (after six months following initial discharge) was 5.9 days/patient-yr in the 'risk' group compared

\begin{tabular}{|c|c|c|c|c|}
\hline & \multicolumn{2}{|l|}{ Univariate analysis } & \multicolumn{2}{|c|}{ Multivariable analysis } \\
\hline & $\mathrm{RR}(95 \% \mathrm{CL})$ & $p$-value & RR $(95 \%$ CL) & $\mathrm{p}$-value \\
\hline \multicolumn{5}{|l|}{ Early period } \\
\hline \multicolumn{5}{|l|}{ Time to rejection } \\
\hline Risk group & $1.16(0.33-4.14)$ & 0.82 & $0.74(0.18-3.12)$ & 0.68 \\
\hline Race (Black) & $5.13(1.06-24.39)$ & 0.04 & $5.38(0.93-31.25)$ & 0.06 \\
\hline Age at transplant $(>10 \mathrm{yr})$ & $1.34(0.40-4.46)$ & 0.63 & $1.33(0.35-5.09)$ & 0.68 \\
\hline \multicolumn{5}{|l|}{ Hospital days } \\
\hline Risk group & $1.49(1.10-2.02)$ & 0.01 & $1.29(0.94-1.77)$ & 0.12 \\
\hline \multicolumn{5}{|l|}{ Low $\mathrm{Cl}$ levels } \\
\hline Risk group & $2.80(1.32-5.94)$ & 0.007 & $2.93(1.32-6.48)$ & 0.008 \\
\hline \multicolumn{5}{|l|}{ Late period } \\
\hline \multicolumn{5}{|l|}{ Time to rejection } \\
\hline Risk group & $9.10(2.68-30.92)$ & $<0.001$ & $3.40(0.95-12.14)$ & 0.06 \\
\hline Race (Black) & $14.49(1.88-90.91)$ & 0.01 & $10.1(2.55-40)$ & 0.001 \\
\hline Age at transplant $(>10 \mathrm{yr})$ & $12.37(2.63-58.18)$ & 0.002 & $7.32(1.13-47.28)$ & 0.04 \\
\hline \multicolumn{5}{|l|}{ Hospital days } \\
\hline Risk group & $1.56(1.24-1.97)$ & 0.002 & $3.14(2.37-4.15)$ & $<0.001$ \\
\hline \multicolumn{5}{|l|}{ Low $\mathrm{Cl}$ levels } \\
\hline Risk group & $3.23(1.90-5.47)$ & $<0.001$ & $2.92(1.52-5.60)$ & 0.001 \\
\hline
\end{tabular}

Table 1. Relative risk for time to rejection, all-cause hospitalization and proportion of outpatient trough calcineurin levels $<50 \%$ target

$\mathrm{RR}$, relative risk; $\mathrm{CL}$, confidence limits; $\mathrm{Cl}$, calcineurin inhibitor. 
with 3.7 days/patient-yr in controls $(\mathrm{p}<0.001)$ (Table 1). After adjusting for patient age and race in the multivariable analysis, there was no significant difference between the 'risk' and control groups for all-cause hospital stay during the early period (RR for 'risk' group 1.29, 95\% confidence intervals 0.94-1.77). However, during the late period, patients in the 'risk' group had a longer all-cause hospital stay even after adjusting for age and race (RR for 'risk' group 3.14, 95\% confidence intervals 2.37-4.15).

\section{$\mathrm{Cl}$ levels}

The 'risk' group had a significantly greater proportion of all CSA or FK levels that were $<50 \%$ of the target levels (defined as the midtherapeutic range level) during both (early and late) time periods (Table 1). During the first six months (early time period), $8.9 \%$ of all levels in the 'risk' group and $3.4 \%$ of all levels in the control group were $<50 \%$ of the target levels $(\mathrm{p}=0.007)$. During the late period (after six months), $14.8 \%$ of all levels in the 'risk' group and $5.1 \%$ of all levels in controls were $<50 \%$ of the target levels $(\mathrm{p}<0.0001)$.

\section{Discussion}

Although parental psychosocial evaluation is routinely performed during pretransplant assessment of pediatric recipients, this study describes for the first time, an association between parental pretransplant psychosocial evaluation and posttransplant recipient morbidity in pediatric heart transplant recipients. After the first six months of follow up, children of parents assigned to psychosocial 'risk' category were 3.4 times more likely to have a rejection episode and 3.1 times more likely to be inpatient compared with controls. These findings suggest that the patients in the psychosocial 'risk' category may benefit from closer outpatient monitoring and a higher level of psychosocial support than practiced currently.

Patients in the psychosocial 'risk' category were 2.9 times more likely to have low trough CSA or FK levels during both early and late follow-up periods. Despite more frequent subtherapeutic levels in the 'risk' group, a higher rejection rate was not noted in these patients during the 'early' period. This may be due to frequent dose adjustments during the early period in response to these low levels. Because patients are monitored less frequently during the 'late' period, subtherapeutic levels may persist for a long enough duration as to increase the likelihood of acute rejection.
Although we did not assess compliance by any other method, noncompliance could potentially explain the finding of more frequent subtherapeutic levels as well as higher rejection-risk in these patients. Non-compliance has been reported to be as high as $33 \%$ in some patient cohorts (9). In the current era of OHT, immune suppression is lifelong and electronic event monitoring has demonstrated that patients who had even minor variations in their dosing schedule were at higher risk for late acute rejection episodes (10). High variability in trough CSA levels has been demonstrated to be a marker for pediatric OHT recipients at greater risk for recurrent rejection and hospitalization (11). Therefore, compliant administration of immunosuppressants must be ensured to decrease the incidence of transplant-related complications.

Identifying at-risk children for transplantrelated morbidity based on caregivers' psychosocial evaluation is consistent with previous studies that have correlated these evaluations in adult recipients with their own medical outcome (12). In adult OHT recipients, psychosocial variables have been found to be associated with compliance, number of rejection episodes and the development of coronary lesions (7). Socio-demographic factors including income, geographic location and stability of the family were shown to be significant predictors of graft survival in renal transplant recipients (12). Stratifying patients into categories depending on the level of psychological symptoms, emotional stability, and a history of noncompliance to therapy has been useful to predict outcome $(13,14)$. Previous studies have also found an association between focused psychological inventories that assess specific personality parameters and poor clinical outcome (13, 15). In a pediatric cohort, a correlation between pretransplant and post-transplant psychological functioning was found in patients and their families following OHT. However, pretransplant psychosocial data was not correlated with medical outcome parameters in that study (16).

We found black race to be an important risk factor for rejection both during early and late periods following hospital discharge. This data supports previously published reports of higher morbidity and worse survival in blacks in both children and adults $(3,4,17,18)$. Furthermore, our study confirmed a previously published finding that teenage age (age $>10 \mathrm{yr}$ in our study) is an independent predictor of rejection. 


\section{Limitations}

The major limitation of this study is a small sample size (number of patients). The duration of follow up and inclusion of multiple events (all rejections and all hospitalizations) for all patients resulted in sufficient number of outcome events for comparison of the two groups. Recognizing the difficulty in adjusting for too many variables with a small sample size, we chose to adjust for only two previously established risk factors, race and age and to treat each of these two variables as dichotomous variables (black vs. non-black rather than the actual race; $>10$ vs. $\leq 10 \mathrm{yr}$ rather than age as a continuous variable). The dramatic change noted in the effect size (RR change from 9.1 in univariate analysis to 3.4 after adjustment for age and race) justifies performing this analysis. It improves internal validity of the proposed risk factor at the cost of making the estimate less precise (with wide 95\% confidence intervals). The small sample size limited our ability to: (i) analyze subgroups of 'risk' category patients ('mild' and 'major' risk categories to observe a gradient effect); (ii) adjust for additional covariates in a multivariable analysis; and (iii) examine the interaction between race, age, and risk group; these analyses were not attempted.

A second limitation of the study was that the assignment of risk category in each patient was not based on a quantifiable standardized psychosocial scoring system. This added an element of subjectivity to this classification. We adopted this approach because the pretransplant consultations did not collect data with equal details on all psychosocial domains for all patients. This prevented us from using a scoring system to classify patients into risk categories. We acknowledge the potential problem in directly extrapolating this classification to other programs. Although imprecise, this approach may be useful because the discussion regarding psychosocial risk in clinical settings during the pretransplant work-up is based on a composite of psychosocial information similar to that utilized in this study. Furthermore, the findings of this study should provide an impetus to further define and quantify this risk factor (psychosocial risk) prospectively.

In summary, the results of this study suggest an association between pretransplant parental psychosocial risk assessment and post-transplant medical outcome in children after cardiac transplantation. The association demonstrated in this study is hypothesis generating for a larger prospective study to more clearly define this risk factor using a standardized scoring system applicable across institutions. Such a study may also be helpful in identification of important individual psychosocial variables that may affect post-transplant outcome.

\section{Financial support}

This study was performed without any external financial support.

\section{References}

1. Boucek MM, Edwards LB, Keck BM, et al. The Registry of the International Society for Heart and Lung Transplantation: Sixth Official Pediatric Report-2003. J Heart Lung Transplant 2003: 22: 636-652.

2. Boucek MM, Edwards LB, Keck BM, et al. Registry for the International Society for Heart and Lung Transplantation: Seventh Official Pediatric Report - 2004. J Heart Lung Transplant 2004: 23: 933-947.

3. Chin C, Naftel DC, Singh TP, et al. Risk factors for recurrent rejection in pediatric heart transplantation: A multicenter experience. J Heart Lung Transplant 2004: 23: 178-185.

4. Park MH, Tolman DE, Kimball PM. The impact of race and HLA matching on long-term survival following cardiac transplantation. Transplant Proc 1997: 29: 1460-1463.

5. Espinoza C, Manito N, Castells E, et al. Pretransplant risk factors of early mortality after orthotopic heart transplantation. Transplant Proc 1999: 31: 2507-2508.

6. Favaloro RR, Perrone SV, Moscoloni SE, et al. Value of pre-heart-transplant psychological evaluation: Long-term follow-up. Transplant Proc 1999: 31: 3000-3001.

7. Shapiro PA, Williams DL, Foray AT, et al. Psychosocial evaluation and prediction of compliance problems and morbidity after heart transplantation. Transplantation 1995: 60: 1462-1466.

8. Falkenstein K, Flynn L, Kirkpatrick B, et al. Non-compliance in children post-liver transplant. Who are the culprits? Pediatr Transplant 2004: 8: 233-236.

9. De Geest S, Abraham I, Moons P, et al. Late acute rejection and subclinical noncompliance with cyclosporine therapy in heart transplant recipients. J Heart Lung Transplant 1998: 17: 854-863.

10. Flippin MS, CANTER CE, BALZER DT. Increased morbidity and high variability of cyclosporine levels in pediatric heart transplant recipients. J Heart Lung Transplant 2000: 19: 343-349.

11. Mongeau JG, Clermont MJ, Robitaille P, et al. Study of psychosocial parameters related to the survival rate of renal transplantation in children. Pediatr Nephrol 1997: 11: 542546.

12. Brandwin M, Trask PC, Schwartz SM, et al. Personality predictors of mortality in cardiac transplant candidates and recipients. J Psychosom Res 2000: 49: 141-147.

13. Block AR, OHnmeiss DD, Guyer RD, et al. The use of presurgical psychological screening to predict the outcome of spine surgery. Spine J 2001: 1: 274-282.

14. Phipps L. Psychiatric evaluation and outcomes in candidates for heart transplantation. Clin Invest Med 1997: 20: 388-395.

15. DeMaso Dr, Douglas Kelley S, Bastardi $\mathrm{H}$, et al. The longitudinal impact of psychological functioning, medical severity, and family functioning in pediatric heart transplantation. J Heart Lung Transplant 2004: 23: 473-480.

16. Mills RM, Naftel DC, Kirklin JK, et al. Heart transplant rejection with hemodynamic compromise: A multiinstitutional study of the role of endomyocardial cellular infiltrate. Cardiac 
Pretransplant psychosocial assessment

Transplant Research Database. J Heart Lung Transplant 1997: 16: 813-821.

17. Pamboukian SV, Costanzo Mr, Meyer P, et al. Influence of race in heart failure and cardiac transplantation: Mortality differences are eliminated by specialized, comprehensive care. J Card Fail 2003: 9: 80-86.
18. Webber SA, Naftel DC, Parker J, et al. Late rejection episodes more than 1 year after pediatric heart transplantation: Risk factors and outcomes. J Heart Lung Transplant 2003: 22: 869-875. 